DOI: 10.5586/am.1097

Publication history

Received: 2016-04-27

Accepted: 2017-01-13

Published: 2017-07-28

This article was originally

submitted to and processed by

the Acta Agrobotanica (another

journal of the Polish Botanical

Society) editors. Upon consent

of the authors and the editors of

both journals, it was eventually

published in Acta Mycologica.

Handling editor

Piotr Sugier (Acta Agrobotanica),

Faculty of Biology and

Biotechnology, Maria Curie-

Skłodowska University in Lublin,

Poland

Authors' contributions

AW: research idea, coordination,

and conduct, manuscript

preparation; JM: manuscript

preparation, reviewed drafts of the paper; At: prepared figures and tables, reviewed drafts of the paper

Funding

The research has been conducted on the authors own expenses.

\section{Competing interests}

No competing interests have

been declared.

\section{Copyright notice}

(c) The Author(s) 2017. This is an Open Access article distributed under the terms of the Creative Commons Attribution License, which permits redistribution, commercial and noncommercial, provided that the article is properly cited.

\section{Citation}

Wieczorek A, Łysko A

Motiejŭnaite J. New and interesting species of lichens

from xerothermic habitats in NW Poland. Acta Mycol. 2017:52(1):1097. https://doi. org/10.5586/am.1097

\section{Digital signatur}

This PDF has been certified using digital signature with a trusted timestamp to assure its origin and integrity. A verification trust dialog appears on the PDF document when it is opened in a compatible PDF reader. Certificate properties provide further details such as certification time and a signing reason in case any altertions and a signing reason in case any alterations made to the final content. If the certificate is missing or invalid it is recommended to verify the article on the journal website.
ORIGINAL RESEARCH PAPER

\section{New and interesting species of lichens from xerothermic habitats in NW Poland}

\author{
Anetta Wieczorek ${ }^{1,2 *}$, Andrzej Łysko ${ }^{3}$, Jurga Motiejŭnaite ${ }^{4}$ \\ ${ }^{1}$ Department of Ecology, University of Szczecin, Waska 13, 71-412 Szczecin, Poland \\ ${ }^{2}$ Center for Molecular Biology and Biotechnology, Faculty of Biology, University of Szczecin, \\ Wąska 13, 71-415 Szczecin, Poland \\ ${ }^{3}$ Department of Environmental Protection and Management, Western Pomeranian University of \\ Technology, Słowackiego 17, 71-43 Szczecin, Poland \\ ${ }^{4}$ Laboratory of Mycology, Nature Research Center, Institute of Botany, Žaliuju ežerų 49, 2021 \\ Vilnius, Lithuania \\ *Corresponding author. Email: anetta.wieczorek@usz.edu.pl
}

\begin{abstract}
This paper presents data on the occurrence of lichens in xerothermic grasslands, representing a great mycological peculiarity of the NW part of Pomerania, Poland. The 12 examined specimens of six species originated from fieldwork carried out in 2011-2014 in the nature reserves Brodogóry, Stary Przylep, Bielinek, Wrzosowiska Cedyńskie, Prof. Adam Wodziczko Nature Reserve in the Wolin National Park, and an old chalk excavation site on Wolin Island. Within the study sites, four lichen species were recorded as a new to Western Pomerania: Agonimia gelatinosa, Collema cristatum, Dermatocarpon luridum, and Leptogium subtile. The other two species, Collema auriforme and C. flaccidum, are rarely observed in the studied region.
\end{abstract}

\section{Keywords}

biodiversity; lichens; nature reserves; NW Poland

\section{Introduction}

The great peculiarity of NW Pomerania are a xerothermic grasslands. They develop in specific climatic conditions only, particularly in the areas where high soil and nearsurface air layer temperatures occur periodically. Soils overgrown with xerothermic plants must be reasonably fertile and rich in calcium carbonate. Such conditions create favorable habitats for development of xerothermophilous and calciphilous lichens [1]. In Poland, calciphilous lichens usually occur in the mountains and uplands in limestone areas [2,3]. In lowlands, this group of lichens is rarely recorded in natural ranges of xerothermic grasslands of the Lower Vistula, Odra, and Warta rivers [4-7]. Xerothermic grasslands of NW Poland are found in morainic areas, characterized by varied relief. The vegetation is not dense, composed mostly of patches of the feather-grass community Potentillo-Stipetum capillatae. Because of its loose structure and specific soil conditions, the community also includes many plants typical of sandy grasslands of the class Koelerio glaucae-Corynephoretea canescentis as well as thermophilous and heliophilous species of the class Festuco-Brometea [8,9] (and also unpublished documentation by Dr Tadeusz Głazek from 1971 "Rezerwat florystyczno-dydaktyczny 'Stary Przylep' ”).

Many lichens are adapted to adverse environmental conditions. They may colonize both natural and man-made substrata (such as concrete and bricks), and are often found in anthropogenic habitats, like wooden constructions, mechanically disturbed soils, worked stone surfaces and quarries. Xerothermic lichens in northern Poland are reported with increasing frequency in anthropogenic habitats, such as gravel pits, mine workings or quarries [10-12]. Such moderate anthropogenic pressure creates new habitats for lichens and may significantly increase their diversity.

This paper presents new localities of lichens that were recorded in xerothermic habitats in NW Poland, developed partly as a result of human activity. 


\section{Material and methods}

The presented list of records is derived from the material collected during fieldwork in 2011-2014. The study included the following study sites: Brodogóry Nature Reserve (B), Stary Przylep Nature Reserve (SP), Prof. Adam Wodziczko Nature Reserve (AW) in the Wolin National Park, an old chalk (OC) excavation site on Wolin Island, Bielinek Nature Reserve (BI), and Wrzosowiska Cedyńskie Nature Reserve (WC) (Fig. 1). In total, 12 specimens of six species were collected, now deposited in the Lichen Herbarium (SZUB-L) of the Department of Ecology and Environmental Protection at the University of Szczecin. Morphological and anatomical observations were made using standard microscopic techniques, under an inverted fluorescence microscope (Zeiss Axio Observer A1) and a light microscope (Zeiss Axio Scope A1).

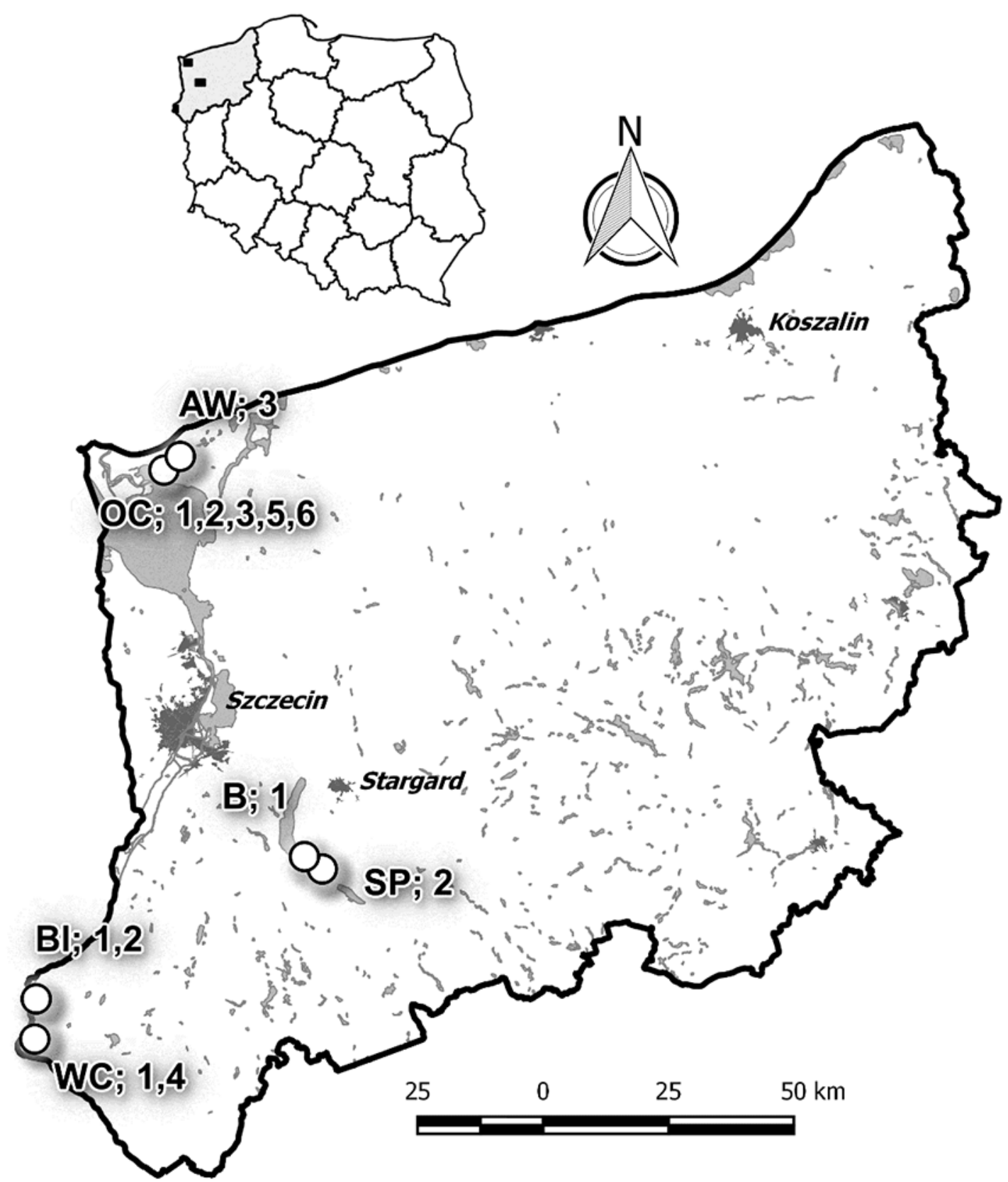

Fig. 1 Distribution map of the study sites, with numbers of the listed species found. AW - Prof. Adam Wodziczko Nature Reserve in the Wolin National Park; OC - old chalk excavation site on Wolin Island; B - Brodogóry Nature Reserve; SP - Stary Przylep Nature Reserve; WC - Wrzosowiska Cedyńskie Nature Reserve; BI - Bielinek Nature Reserve; 1 - Agonima gelatinosa; 2 - Collema auriforme; 3 - C. cristatum; 4 - C. flacidium; 5 - Dermatocarpon luridum; 6 - Leptogium subtile. 


\section{Results: list of recorded species}

Agonima gelatinosa (Ach.) Brand \& Diederich (Fig. 2a)

Terricolous (found on calcareous soil), muscicolous and also on plant debris. New to NW Poland. This rare species in Poland is known from the Gorce Mts [13,14], Kujawy region [7,15,16], Przemyśl town [17], Sudety Mts [18], and Tatry Mts [19].

Specimens examined. Old chalk excavation site on Wolin Island (N 5353'08.1" / E $\left.14^{\circ} 27^{\prime} 11.7^{\prime}\right)$ on soil, leg. Anetta Wieczorek, Andrzej Łysko, SZUB-L 3008; Wrzosowiska Cedyńskie Nature Reserve (N 52 51'16.1" / E 14²10'27.3'), on soil, leg. Anetta Wieczorek, Andrzej Łysko, SZUB-L 3009; Bielinek Nature Reserve (N 5255'24.7" / E 14¹0'18.1'), on soil, leg. Anetta Wieczorek, Andrzej Łysko, SZUB-L 3007; Brodogóry Nature Reserve (N 53⒉ $1^{\prime} 32.7^{\prime \prime} /$ E $\left.14^{\circ} 93^{\prime} 08^{\prime \prime}\right)$, on soil, leg. Anetta Wieczorek, Andrzej Łysko, SZUB-L 3010 .

\section{Collema auriforme (With.) Coppins \& J. R. Laundon (Fig. 2b)}

In Pomerania recorded earlier at one site in Bory Tucholskie forest [20,21]. It is also reported from southern Poland, e.g., the Beskid Mały Mts [22], Beskid Sądecki Mts [23], Beskid Śląski Mts [24], Beskid Wyspowy Mts and Beskid Żywiecki Mts [25], Stołowe Mts [26], Kielce town [27], Kraków City [28], Pieniny Mts [29], Spiskie Foothills [30], Rożnowskie Foothills [31], Sudety Mts [32-34], Tatry Mts [35-38], Tarnów town [39], near Warsaw [40], and Kraków-Częstochowa Upland [41].

Specimens examined. Old chalk excavation site on Wolin Island (N 53 $53^{\prime} 08.1^{\prime \prime}$ / E $\left.14^{\circ} 27^{\prime} 11.7^{\prime \prime}\right)$, found on mosses, calcareous soil, in moist and shaded conditions, in anthropogenic habitats. It develops characteristic isidia in brownish-green lobes, leg. Anetta Wieczorek, Andrzej Łysko, SZUB-L 3006; Bielinek Nature Reserve (N 52 55'24.7" / E $\left.14^{\circ} 10^{\prime} 18.1^{\prime \prime}\right)$, on calcareous soil in natural habitats, leg. Anetta Wieczorek, Andrzej Łysko, SZUB-L 3004; Stary Przylep Nature Reserve (N 53¹9'08" / E 1499'41"), on calcareous soil, leg. Anetta Wieczorek, Andrzej Łysko, SZUB-L 2996.

\section{Collema cristatum (L.) Weber in F. H. Wigg. (Fig. 2c)}

Within Poland reported previously only from the southern part of the country, e.g., the Beskidy Zachodnie Mts [42], Beskid Sądecki Mts [23], Vistula valley [43], Świętokrzyskie Mts [44], Kielce town [27], Kraków City [28], Chęciny region [45], Pieniny Mts [3,29], Śląsk Opolski town [46], Tatry Mts [37,47,48], Kraków-Częstochowa Upland [41], and Wieluńska Upland [49].

Specimens examined. Prof. Adam Wodziczko Nature Reserve in the Wolin National Park (N 5351'50.0" / E 14²7'06.0'), on calcareous soil on sun-exposed sites in natural habitats, leg. Anetta Wieczorek, Andrzej Łysko, SZUB-L 3003; old chalk excavation

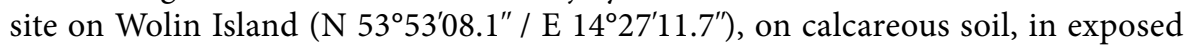
conditions, in artificial (excavation) habitats, leg. Anetta Wieczorek, Andrzej Łysko, SZUB-L 3002.

\section{Collema flaccidum (Ach.) Ach. (Fig. 2d)}

In Poland earlier known from several localities in the north of the country and several in the south [50]. Reported in the north of Kaszubskie Coastland [20,51,52], Suwałki Landscape Park [53], and near Białowieża National Park [54]. In the south of the country reported from the Bieszczady Mts [55,56], Beskid Niski Mts [57], Beskid Mały Mts [22], Beskid Sądecki Mts [23,58,59], Beskid Średni Mts [60], Beskid Wyspowy Mts and Beskid Żywiecki Mts [61], Gorce Mts [62], Stołowe Mts [26], Sudety Mts [63], and Tatry Mts $[35,37,64,65]$. 

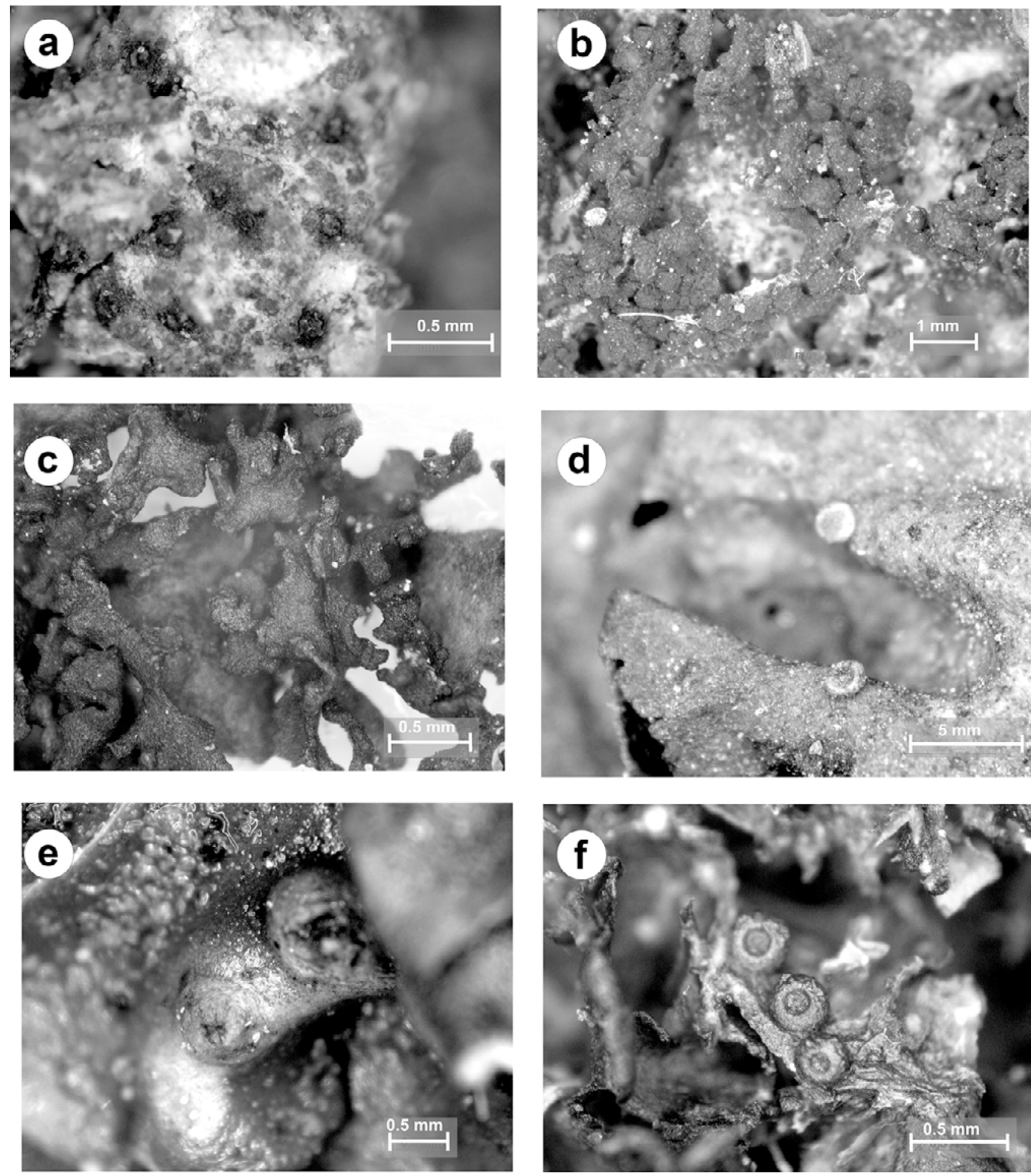

Fig. 2 Thalli of xerothermic lichens. 1 - Agonima gelatinosa; 2 - Collema auriforme; 3 - C. cristatum; 4 - C. flacidium; 5 - Dermatocarpon luridum; 6 - Leptogium subtile. 
Specimens examined. Wrzosowiska Cedyńskie Nature Reserve (N 5251'16.1" / E $14^{\circ} 10^{\prime} 27.3^{\prime \prime}$ ), on damp rock, among mosses in open habitats, on the edge of the reserve, leg. Anetta Wieczorek, Andrzej Łysko, SZUB-L 3001.

\section{Dermatocarpon luridum (With.) J. R. Laundon (Fig. 2e)}

The record from the old chalk excavation site is the first record from northern Poland. Previously reported from only five localities in southern Poland, e.g., the Bieszczady Mts [55,56], Beskid Wyspowy Mts and Beskid Żywiecki Mts [61], Lower Silesia [66], Sudety Mts [63], and Tatry Mts [37,38,65,67-70].

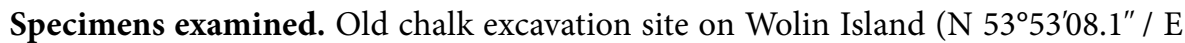
$14^{\circ} 27^{\prime} 11.7^{\prime \prime}$ ), grows on exposed calcareous soil around the margins of water bodies, leg. Anetta Wieczorek, Andrzej Łysko, SZUB-L 3011.

\section{Leptogium subtile (Schrad.) Torss. (Fig. 2f)}

New to northern Poland. Previously reported from only two localities in southern Poland: Bielska Plain [71] and the Gorce Mts [62].

Specimens examined. Old chalk excavation site on Wolin Island (N 53 $53^{\prime} 08.1^{\prime \prime}$ / E $\left.14^{\circ} 27^{\prime} 11.7^{\prime \prime}\right)$, on debarked wood and mosses in humid places, leg. Anetta Wieczorek, Andrzej Łysko, SZUB-L 2998.

\section{Discussion}

In the studied localities, xerothermic epigeic lichens were found on calcareous soil, humus, and decaying remains of plants as well as on rock-waste. Calciphilous lichens were not homogeneous with respect to specific habitat requirements. Here, a common factor was only the chemical nature of substratum (calcium carbonate). Other factors differed, e.g., the degree of sun exposure or substratum moisture [72].

In total, six new species of lichenized fungi were observed: one in the Prof. Adam Wodziczko Nature Reserve in the Wolin National Park, five in the old chalk excavation site on Wolin Island, one in the Brodogóry Nature Reserve, one in the Stary Przylep Nature Reserve, two in the Wrzosowiska Cedyńskie Nature Reserve, and two in the Bielinek Nature Reserve (Fig. 1). The species are either new or rare in Western Pomerania. All the collected lichens were found in isolated localities, in small populations. Their thalli were healthy, with well-developed reproductive organs, and did not show any symptoms of decline. Four of the recorded lichens were new to NW Pomerania: Agonimia gelatinosa, Collema cristatum, Dermatocarpon luridum, and Leptogium subtile (Fig. 2). They were found together in the old chalk excavation site located in the buffer zone of the Wolin National Park.

Lichens of xerothermic habitats are usually inconspicuous and rarely observed. They are described chiefly during specialized lichenological explorations. Currently, their distribution in Europe is well-studied. They are recorded in several climatic zones, from the boreal to the Mediterranean zone [73], but most frequently in SE and S parts of Europe with a continental climate. In other parts of the continent, with a more humid climate, isolated patches of steppe vegetation are found on particularly warm and dry sites [74]. The patches occupy calcium-rich, steep riverbanks, ravines, or calcareous rocks. In Central Europe, xerothermic lichens are rare or very rare, particularly in the lowlands [75-78] (Tab. 1). The small size of the patches and the increasing eutrophication of adjacent areas are important threats to those populations. The presented records are some of the northernmost localities of xerothermic lichens, which are particularly important for the dynamics of those taxa. 
Tab. 1 Other European records of the lichen species reported in this study.

\begin{tabular}{|c|c|}
\hline Species & Countries \\
\hline $\begin{array}{l}\text { Agonima gelatinosa (Ach.) M. Brand \& } \\
\text { Diederich }\end{array}$ & Estonia [79], Netherlands [78], Poland [80] \\
\hline $\begin{array}{l}\text { Collema auriforme (With.) Coppins \& J. R. } \\
\text { Laundon }\end{array}$ & $\begin{array}{l}\text { Albania [81], Austria [82], Bosnia and Herzegovina [83], Bulgaria [84], Croatia } \\
\text { [85], France [86], Germany [87], Greece [88,89], Iceland [90], Macedonia [91], } \\
\text { Netherlands [78], Poland [80], Slovakia [92], Slovenia [93,94], Spain }[95,96] \text {, } \\
\text { Switzerland [97-101], Turkey [102-106] }\end{array}$ \\
\hline Collema cristatum (L.) F. H. Wigg. & $\begin{array}{l}\text { Austria [82], Bosnia and Herzegovina [83], Bulgaria [84], Croatia [85], Estonia } \\
\text { [79], Germany [87], Italy [107,108], Ireland [109], Lithuania [110], Poland [80], } \\
\text { Russia [111], Slovenia [94,107], Spain [96,112], Switzerland [101,113,114], Turkey } \\
{[115-123]}\end{array}$ \\
\hline Collema flaccidum (Ach.) Ach. & $\begin{array}{l}\text { Austria [82,124,125], Bosnia and Herzegovina [83], Bulgaria [84], France [126], } \\
\text { Germany [88,127], Greece [89], Latvia [128], Macedonia [91], Poland [80], Ro- } \\
\text { mania [129], Serbia [130], Switzerland [98,101] }\end{array}$ \\
\hline $\begin{array}{l}\text { Dermatocarpon luridum (With.) J. R. } \\
\text { Laundon }\end{array}$ & $\begin{array}{l}\text { Bosnia and Herzegovina [83], Bulgaria [84], Lithuania [131], Estonia [132], } \\
\text { Germany [87], Greece [89], Poland [80], Portugal [133], Spain [134], Switzerland } \\
\text { [135], Turkey [104,136] }\end{array}$ \\
\hline Leptogium subtile (Schrad.) Torss. & $\begin{array}{l}\text { Austria [137], Bosnia and Herzegovina [83], Bulgaria [84], Czech Republic } \\
{[138,139], \text { France [126], Germany [87,140], Netherlands [78], Poland [80], Rus- }} \\
\text { sia [141-144], Spain [145,146], Sweden [147], Switzerland [100,113,148], Turkey } \\
{[123,149] \text {, Ukraine [150] }}\end{array}$ \\
\hline
\end{tabular}

\section{References}

1. Czyżewska K. Flora porostów naziemnych w Załęczańskim Parku Krajobrazowym (Wyżyna Wieluńska). Acta Universitatis Lodzensis, Folia Sozologica. 1986;2:315-341.

2. Tobolewski Z. Z badań nad florą porostów Pienin. Fragmenta Floristica et Geobotanica. 1954;1(2):3-13.

3. Tobolewski Z. Porosty Pienin. Prace Komisji Biologicznej Poznańskiego Towarzystwa Przyjaciół Nauk. 1958;17(5):1-124.

4. Filipek M. Roślinność kserotermiczna okolic Górzycy pod Kostrzyniem nad Odrą. Badania Fizjograficzne nad Polską Zachodnią. 1962;10:205-213.

5. Filipek M. Roślinność kserotermiczna regionu dolnej Odry i Warty. Prace Komisji Biologicznej Poznańskiego Towarzystwa Przyjaciół Nauk. 1974;38:1-109.

6. Tobolewski Z. Materiały do flory porostów północno-zachodniej Polski. Fragmenta Floristica et Geobotanica. 1962;8(1):67-80.

7. Wieczorek A, Schiefelbein U. Lichens of xerothermic grasslands in Western Pomerania (Poland). Plant Divers Evol. 2013;130(3-4):295-302. https://doi.org/10.1127/1869-6155/2013/0130-0074

8. Celiński F. Pontyjskie zbocza koło Grędźca nad jeziorem Miedwie. Chrońmy Przyrodę Ojczystą. 1953;9(3):25-32.

9. Celiński F, Filipek M. Flora i zespoły roślinne leśno-stepowego rezerwatu w Bielinku nad Odrą. Badania Fizjograficzne nad Polską Zachodnią. 1958;4:13-183.

10. Ceynowa-Giełdon M. Kalcyfilne porosty naziemne na Kujawach. Toruń: Wydawnictwo Uniwersytetu Mikołaja Kopernika; 2001.

11. Sągin B. A few remarks on the lichens found on anthropogenic rocky substrata in northern Poland. In: Fałtynowicz W, Latałowa M, Szmeja J, editors. Dynamics and conservation of the Pomeranian vegetation. Gdańsk: Bogucki Wydawnictwo Naukowe; 1997. p. 19-199.

12. Sągin B. Porosty wapiennych podłoży pochodzenia antropogenicznego w Północnej 
Polsce [PhD thesis]. Gdańsk: Katedra Ekologii Uniwersytetu Gdańskiego; 1998.

13. Czarnota P. Some interesting lichens from Gorce Mts (Western Beskidy Mts) new to Poland. Graph Scr. 1998;9:59-61.

14. Czarnota P, Guttová A, Halda JP, Kukwa M, Liška J, Palice Z, et al. Lišajníky zaznamenané počas 13. Jarného stretnutia bryologicko-lichenologickej sekcie ČBS na exkurzii v Tematínskych Vrchoch (Považský Inovec, Slovensko). Bryonora. 2006;38:2639.

15. Ceynowa-Giełdon M. Polyblastia gelatinosa and P. agraria - new species to lichen flora of Poland. Acta Mycol. 1998;33(2):299-307. https://doi.org/10.5586/am.1998.026

16. Ceynowa-Giełdon M. Naziemne gatunki porostów z rodzaju Verrucaria (Lichenes, Verrucariaceae) na Kujawach i niektórych terenach sąsiednich. Fragm Florist Geobot Pol. 1998;5:243-249.

17. Kiszka J. Porosty (Lichenes) oraz warunki bioekologiczne Przemyśla. Arboretum Bolestraszyce. 1999;6:3-86.

18. Kossowska M. Porosty skał wapiennych w Sudetach - analiza geograficzna i ekologiczna [PhD thesis]. Wrocław: Instytut Botaniki Uniwersytetu Wrocławskiego; 1999.

19. Flakus A. Lichenized and lichenicolous fungi from mylonitized areas of the subnival belt in the Tatra Mountains (Western Carpathians). Ann Bot Fenn. 2007;44:427-449.

20. Fałtynowicz W. The lichens of Western Pomerania (NW Poland). An ecogeographical study. Cracow: W. Szafer Institute of Botany, Polish Academy of Sciences; 1992. (Polish Botanical Studies; vol 4).

21. Fałtynowicz W, Kukwa M. Lista porostów i grzybów naporostowych Pomorza Gdańskiego. Acta Botanica Cassubica, Monographiae. 2006;2:1-98.

22. Nowak J. Porosty Beskidu Małego. Fragmenta Floristica et Geobotanica. 1965;11(3):421462.

23. Śliwa L. Antropogeniczne przemiany lichenoflory Beskidu Sądeckiego. Kraków: Instytut Botaniki Uniwersytetu Jagiellońskiego; 1998. (Prace Botaniczne; vol 31).

24. Kiszka J. Porosty Beskidu Śląskiego. Rocznik Naukowo-Dydaktyczny. Wyższa Szkoła Pedegogiczna w Krakowie. 1967;28:5-91.

25. Nowak J. Porosty Beskidów Wyspowego i Żywieckiego, Pasma Jałowca i Masywu Babiej Góry. Łódź: Polskie Towarzystwo Botaniczne; 1998. (Monographiae Botanicae; vol 83). https://doi.org/10.5586/mb.1998.002

26. Tobolewski Z. Porosty Gór Stołowych. Prace Komisji Biologicznej Poznańskiego Towarzystwa Przyjaciół Nauk. 1955;16(1):1-100.

27. Toborowicz K. Porosty miasta Kielc i najbliższej okolicy. Fragmenta Floristica et Geobotanica. 1976;22(4):574-603.

28. Kiszka J, Kościelniak R. Porosty miasta Krakowa oraz waloryzacja ich warunków bioekologicznych. Studia Ośrodka Dokumentacji Fizjograficznej. 1996;24:21-73.

29. Kiszka J. Porosty (Lichenes) dna i otoczenia zbiorników retencyjnych w dolinie Dunajca w Pieninach. Fragm Florist Geobot Pol. 1997;4:253-323.

30. Kiszka J. Porosty Pogórza Spiskiego. Studia Ośrodka Dokumentacji Fizjograficznej. 1985;13:213-243.

31. Kozik R. Porosty (Lichenes) Pogórza Rożnowsko-Ciężkowickiego. Fragmenta Floristica et Geobotanica. 1977;23(2):215-252.

32. Szczepańska K, Kossowska M. Porosty Doliny Kleśnicy w Masywie Śnieżnika (Sudety Wschodnie). Acta Botanica Silesiaca. 2003;1:177-187.

33. Kossowska M, Fabiszewski J. Threatened lichens of Lower Silesia, Poland. Acta Soc Bot Pol. 2004;73(2):139-150. https://doi.org/10.5586/asbp.2004.020

34. Szczepańska K. Antropogeniczne przemiany bioty porostów Masywu Śnieżnika i Gór Bialskich. Acta Botanica Silesiaca Monographiae. 2008;4:1-294.

35. Motyka J. Die Pflanzenassoziationen des Tatra-Gebirges. II Teil: Die epilitischen Assoziationen der nitrophilen Flechten im Polonischen Teile der Westtatra. Bulletin de l'Academie Polonaise des Sciences et des Lettres, Classe des Mathématiques et Naturelles, Série B, Sciences Naturelles. 1924;9(10):835-850.

36. Kiszka J. Porosty pasma Gubałówki. Fragmenta Floristica et Geobotanica. 1967;13(3):419-446.

37. Alstrup V, Olech M. Checklist of the lichens of the Tatra National Park, Poland. Zeszyty 
Naukowe Uniwersytetu Jagiellońskiego. Prace Botaniczne. 1992;24:185-206.

38. Bielczyk U. Materiały do flory porostów Tatr ze zbiorów Muzeum Tatrzańskiego. Fragm Florist Geobot Pol. 1997;4:329-343.

39. Kozik R. Porosty miasta Tarnowa i okolicy. Fragmenta Floristica et Geobotanica. 1970;16(2):361-381.

40. Filipowicz K. Spis mchów, wątrobowców i porostów z niektórych stanowisk Królestwa Polskiego, a mianowicie z Doliny Ojcowskiej i Bentkowickiej, okolic Warszawy, Łukowa, Puław i Brześcia Litewskiego, zebranych i oznaczonych w latach 1877 i 1879. Pamiętnik Fizyograficzny. 1884;1:258-267.

41. Nowak J. Porosty Wyżyny (Jury) Krakowsko-Częstochowskiej. Warszawa: Państwowe Wydawnictwo Naukowe; 1961. (Monographiae Botanicae; vol 11). https://doi.org/10.5586/mb.1961.001

42. Nowak J. Materiały do flory porostów Beskidów Zachodnich. 1. Porosty pasma Policy. Fragmenta Floristica et Geobotanica. 1967;13(1):107-139.

43. Kiszka J, Betleja L. Porosty (Lichenes) doliny Wisły. Studia Ośrodka Dokumentacji Fizjograficznej. 1989;17:209-234.

44. Cieśliński S. Porosty Gór Świętokrzyskich. Kielce. Zeszyty Przyrodnicze. 1973;1:1-30.

45. Toborowicz K. Porosty Obszaru Chęcińskiego w Górach Świętokrzyskich. Fragmenta Floristica et Geobotanica. 1983;29(1):121-188.

46. Leśniański G. The lichen collection from Opole Silesia (Poland) housed in the Wrocław University Herbarium. Fragmenta Floristica et Geobotanica. 1999;44(1):141-149.

47. Tobolewski Z. Materiały do flory porostów Tatr. Prace Komisji Biologicznej Poznańskiego Towarzystwa Przyjaciół Nauk. 1956;17(2):1-33.

48. Tobolewski Z. Lichenotheca Polonica. Fasc. VII, No. 126-150. Lichenes Tatrenses. Poznań: Wyd. PAN; 1956. p. 9.

49. Nowak J. Porosty Wyżyny Wieluńskiej. Acta Mycol. 1967;3:209-242. https://doi.org/10.5586/am.1967.012

50. Fałtynowicz W. Collema flaccidum (Ach.) Ach. In: Cieśliński S, Fałtynowicz W, editors. Atlas of the geographical distribution of lichens in Poland. Part II. Cracow: W. Szafer Institute of Botany, Polish Academy of Sciences; 1999. p. 13-17.

51. Krawiec F. Flora epilityczna głazów narzutowych zachodniej Polski. Prace Komisji Matematyczno-Przyrodniczej Poznańskiego Towarzystwa Przyjaciół Nauk. 1938;9(2):1254.

52. Fałtynowicz W. Porosty północno-zachodniej części Borów Tucholskich. Fragmenta Floristica et Geobotanica. 1980;26(1):81-102.

53. Kukwa M, Fałtynowicz W. Porosty i grzyby naporostowe rezerwatu "Głazowisko Bachanowo nad Czarną Hańczą" i terenów przyległych w Suwalskim Parku Krajobrazowym (NE Polska). Parki Narodowe i Rezerwaty Przyrody. 2002;21(4):375384.

54. Cieśliński S. Atlas rozmieszczenia porostów (Lichenes) w Polsce północno-wschodniej. Warszawa: Białowieska Stacja Geobotaniczna UW; 2003. (Phytocoenosis Supplementum Cartographiae Geobotanicae; vol 15).

55. Glanc K, Tobolewski Z. Porosty Bieszczadów Zachodnich. Prace Komisji Biologicznej Poznańskiego Towarzystwa Przyjaciół Nauk. 1960;21(4):1-108.

56. Kiszka J, Kościelniak R. The flora of lichens in the Polish Eastern Carpathians. In: Kondratyuk S, Coppins B, editors. Lobarion lichens as indicators of primeval forests of the Eastern Carpathians (Darwin International Workshop); 1998 May 25-30; Kostrino, Ukraine. Kiev: M. H. Kholodny Institute of Botany, Ukrainian Phytosociological Center; 1998. p. 82-101.

57. Olech M. Materiały do flory porostów Beskidu Niskiego. Zeszyty Naukowe Uniwersytetu Jagiellońskiego. Prace Botaniczne. 1974;2:181-200.

58. Olech M. Porosty Beskidu Sądeckiego. Zeszyty Naukowe Uniwersytetu Jagiellońskiego. Prace Botaniczne. 1973;1:87-192.

59. Czarnota P. Flora porostów rezerwatu "Żebracze” w Beskidzie Sądeckim. Parki Narodowe i Rezerwaty Przyrody. 2002;21(4):385-410.

60. Nowak J. Porosty Beskidu Średniego (Makowskiego). Cz. 1. Porosty Pasma Pewelskiego oraz wzniesień Lasku i Soliska. Acta Mycol. 1968;4(1):147-174. https://doi.org/10.5586/am.1968.010 
61. Nowak J. Porosty Beskidów Wyspowego i Żywieckiego, Pasma Jałowca i Masywu Babiej Góry. Łódź: Polskie Towarzystwo Botaniczne; 1998. (Monographiae Botanicae; vol 83). https://doi.org/10.5586/mb.1998.002

62. Czarnota P. Porosty Gorczańskiego Parku Narodowego. Część I. Wykaz i rozmieszczenie gatunków. Parki Narodowe i Rezerwaty Przyrody. 2000;19(1):3-73.

63. Fabiszewski J. Porosty Śnieżnika Kłodzkiego i Gór Bialskich. Warszawa: Państwowe Wydawnictwo Naukowe; 1968. (Monographiae Botanicae; vol 26).

64. Tobolewski Z. Materiały do flory porostów Tatr. IV. Prace Komisji Biologicznej Poznańskiego Towarzystwa Przyjaciół Nauk. 1960;21(5):1-31.

65. Alstrup V, Olech M. Additions to the lichen flora of the Polish Tatra Mountains. II. Zeszyty Naukowe Uniwersytetu Jagiellońskiego. Prace Botaniczne. 1990;21:211-217.

66. Kossowska M, Turzańska M. Nowe stanowisko Dermatocarpon luridum (Lichenes, Dermatocarpaceae) na Dolnym Śląsku. Acta Universitatis Wratislaviensis. Prace Botaniczne. 1998;74:5-8.

67. Motyka J. Studia nad florą porostów tatrzańskich. Część 1. Porosty zebrane w Dolinie Kościeliskiej. Acta Societatis Botanicorum Poloniae. 1924;2(1):44-59. https://doi.org/10.5586/asbp.1924.004

68. Motyka J. Die Pflanzenassoziationen des Tatra-Gebirges. VI Teil: Studien über epilitischen Flechtengesellschaften. Bulletin de l'Academie Polonaise des Sciences et des Lettres, Classe des Mathématiques et Naturelles, Série B, Sciences Naturelles. 1926;3(4):189-227.

69. Tobolewski Z. Nowe i rzadkie gatunki we florze porostów Tatr polskich. Prace Komisji Biologicznej Poznańskiego Towarzystwa Przyjaciół Nauk. 1955;17(1):1-36.

70. Bielczyk U. Materiały do geograficznego rozmieszczenia porostów (Lichenes) w Polsce. 1. Porosty Tatr. Fragm Florist Geobot Pol. 1999;6:245-253.

71. Cieśliński S. Porosty muraw kserotermicznych na kemach w północnej części Równiny Bielskiej. Fragmenta Floristica et Geobotanica. 1986;29(3-4):437-449.

72. Kossowska M. Naziemne porosty kalcyfilne w Sudetach. Kalkliebende Bodenflechten in den Sudeten. Przyroda Sudetów Zachodnich. 2003;6:93-100.

73. Wirth V, Hauck M, Schultz M. Die Flechten Deutschlands, Teil 1 \& 2. Stuttgart: Ulmer; 2013.

74. Cwener A. Polskie "stepy”. Dzikie Życie [Internet]. 2006 [cited 2017 Jul 26];10(148). Available from: http://dzikiezycie.pl/archiwum/2006/pazdziernik-2006/polskie-stepy

75. Liška J, Palice Z, Slavíková Š. Checklist and red list of lichens of the Czech Republic. Preslia. 2008;80:151-182.

76. Litterski B, Schiefelbein U. Rote Liste der Flechten Mecklenburg-Vorpommerns. Schwerin: Ministerium für Landwirtschaft, Umwelt und Verbraucherschutz Mecklenburg-Vorpommern; 2007. (Rote Listen der in Mecklenburg-Vorpommern gefährdeten Pflanzen und Tiere).

77. Hauck M, de Bruyn U. Rote Liste und Gesamtartenliste der Flechten in Niedersachsen und Bremen. Hannover: Niedersächsischer Landesbetrieb für Wasserwirtschaft, Küstenund Naturschutz; 2010. (Informationsdienst Naturschutz Niedersachsen).

78. Aptroot A, Herk CM, Sparrius LB. Basisrapport voor de Rode Lijst Korstmossen. OudeTonge: Bryologische en Lichenologische Werkgroep; 2011. (BLWG-rapport; vol 12).

79. Suija A, Czarnota P, Himelbrant D, Kowalewska A, Kukwa M, Kuznetsova E, et al. The lichen biota of three nature reserves in island Saaremaa, Estonia. Folia Cryptogam Est. 2010;47:85-96.

80. Cieśliński S, Czyżewska K, Fabiszewski J. Red list of the lichens in Poland. In: Mirek Z, Zarzycki K, Wojewoda W, Szeląg Z, editors. Red list of plants and fungi in Poland. Cracow: W. Szafer Institute of Botany, Polish Academy of Sciences; 2006. p. 73-89.

81. Hafellner J, Kashta L. Miscellaneous records of lichenes and lichenicolous fungi from Albania. Herzogia. 2003;16:135-142.

82. Hafellner J. Zur Flechtendiversität in den Gurktaler Alpen (Österreich: Kärnten Steiermark und Salzburg). Herzogia. 2005;1:79-138.

83. Bilovitz PO, Stešević D. Epiphytic lichens and lichenicolous fungi from the northern part of Montenegro. Herzogia. 2010;23:249-256. https://doi.org/10.13158/heia.23.2.2010.249

84. Ivanov D. Checklist of the lichens and lichenicolous fungi from the Pirin Mountains in Bulgaria. Berichte des Naturwissenschaftlich-Medizinischen Vereins in Innsbruck. 
2010;96:35-57.

85. Ozimec S, Bošković I, Florijančić T, Dinko Jelkić D, Opačak A, Puškadija Z, et al. The lichen flora of Risnjak National Park (Croatia). Acta Bot Croat. 2010;69(1):19-29.

86. Diederich P, van den Broeck D, Ertz D, Signoret J, Aptroot A, Sparrius L, et al. Contribution to the knowledge of lichens in northern France. Bull Soc Nat Luxemb. 2006;106:53-62.

87. Wirth V, Hauck M, Brackel WV, Cezanne R, de Bruyn U, Dürhammer O, et al. Checklist of lichens and lichenicolous fungi in Germany [Internet]. 2011 [cited 2017 Jun 2]. Available from: http://wwwuser.gwdg.de/ mhauck/02Lichens.pdf

88. Christensen SN, Pisút I, Sipman HJM. New and noteworthy lichen records from the Ionian island of Kerkira (Corfu), Greece. Willdenowia; 1997;27(1-2):265-272. https://doi.org/10.3372/wi.27.2726

89. Christensen SN. The epiphytic lichen flora of Platanus orientalis stands in Greece. Willdenowia. 2014;44(2):209-227. https://doi.org/10.3372/wi.44.44203

90. Seaward MRD, Richardson DHS. Lichens of Lambay Island. Glasra. 2000;4:1-6.

91. Mayrhofer H, Maliček J, Rohrer A, Svoboda D, Bilovitz PO. New or other wise interesting lichenized and lichenicolous fungi from Macedonia. Fritschiana (Graz). 2012;71:1-35.

92. Pišút I, Lackovičová A, Guttová A, Palice Z. New lichen records from Bukovské vrchy Mts (NE Slovakia). Acta Mycol. 2007;42(2):267-280. https://doi.org/10.5586/am.2007.030

93. Bilovitz PO, Batic F, Mayrhofer H. Epiphytic lichen mycota of the virgin forest reserve Rajhenavski Rog (Slovenia). Herzogia. 2011;24:315-324. https://doi.org/10.13158/heia.24.2.2011.315

94. Wllfling A, Mayrhofer H. Contributions to the lichen flora of Slovenia IX. Lichenized and lichenicolous fungi from Crni Kal (Kras). Stapfia. 2002;80:293-310.

95. Zozayai C, Etayo J. Líquenes saxícolas y hongos liquenícolas de la sierra de ujué (Navarra). Stvdia Botánica. 1994;13:263-266.

96. Longán A, Llop E, Navarro-Rosinés P. Aproximació a la flora liquènica de la vall d'Alinyà. In: Germain J, editor. Els sistemes naturals de la vall d’Alinyà. Barcelona: Institució Catalana d'Història Natural; 2004. p. 197-222. (Treballs de la Institució Catalana d'Història Natural; vol 14).

97. Bürgi-Meyer K. Chaenotheca cinerea (Pers.) Tibell: Ein weiterer Fund in der Schweiz. Meylania. 2005;34:18-20.

98. Dietrich M, Bürgi-Meyer K. Interessante Flechten aus dem Kanton Luzern - Neufunde für die Schweiz. Meylania. 2010;45:10-16.

99. Vonarburg C, Keller C, Mermilliod JC, Stofer S. Lichenologische Beobachtungen an der Exkursion der Bryolich ins Wengital, Kt. St. Gallen. Meylania. 2011;46:4-7.

100. Vust M. Les lichens terricoles de Suisse. Lausanne: Société Vaudoise des Sciences Naturelles; 2011. (Mémoires de la Société Vaudoise des Sciences Naturelles; vol 24).

101. Dietrich M, Bürgi-Meyer K. Die Chestenweid am Vierwaldstättersee (Kanton Luzern, Zentralschweiz) ein bedeutender Lebensraum für Flechten trockenwarmer Standorte auf der Alpennordseite. Herzogia. 2011;24(1):33-52. https://doi.org/10.13158/heia.24.1.2011.33

102. John V, Nimis PL. Lichen flora Amanos mountain and the province of Hatay. Turk J Botany. 1998;22:257-267.

103. Yazici K, Aslan A. Additional lichen records from Rize Province. Turk J Botany. 2002;26:181-193.

104. Yazici K, Aptroot A, Aslan A. Lichen biota of Zonguldak, Turkey. Mycotaxon. 2007;102:257-260.

105. Yazici K, Aptroot A, Etayo J, Aslan A, Guttova A. Lichens from the Batman, Mardin, Osmaniye, and Sivas regions of Turkey. Mycotaxon. 2008;103:141-144.

106. Karagoz Y, Aslan A. Floristic lichen records from Kemaliye District (Erzincan) and Van Province. Turk J Botany. 2012;36:558-565.

107. Breuss O. Neue Funde pyrenocarper Flechten aus den Julischen Alpen (Slowenien und Italien). Herzogia. 2008;21:85-92.

108. Nascimbene J. A lichen surrey in the western Dolomites: Schlern Nature Park (S Tyrol NE Italy). Grealeriana. 2008;8:75-94. 
109. Fox HF. Census catalogue of the lichenicolous fungi of Ireland. Dublin: National Botanic Gardens; 2001

110. Motiejūnaitė J. Additions to the Lithuanian flora of foliose and fruticose lichens. Bot Lith. 2002;8(1):69-76.

111. Paukov AG. The lichen flora of serpentine outcrops in the Middle Urals of Russia. Northeast Nat (Steuben). 2009;16(5):341-350. https://doi.org/10.1656/045.016.0525

112. Etayo J. Algunos hongos liquenícolas del Pirineo Aragonés. Studia Botánica. 1994;13:255-257.

113. Vust M, Truong C, Mermilliod JC. Lichens du Vallon de Nant (Bex, Alpes vaudoises). Mémoires de la Société Vaudoise des Sciences Naturelles. 2009;23:51-74.

114. Çobanoğlu G, Akdemįr B. Contribution to the lichen diversity of nature parks in Bolu and Çorum, Anatolia, Turkey. Herzogia. 2004;17:129-136.

115. Öztürk S, Güvenc S, Aydin S. Floristic lichen records from Isparta and Burdur provinces. Turk J Botany. 2005;29:243-250.

116. Halici MG, Aksoy A. Saxicolous and terricolous lichens of fiirvan Mountain (Pinarbasi, Kayseri). Turk J Botany. 2006;30:477-481.

117. Tufan Ö, Sümbül H, Türk AÖ. The lichen flora of the Termessos National Park in Southwestern Turkey. Turkish Lichens. 2006:1-12.

118. Oran S, Öztürk S. Lichen records from Southeast and East Anatolian region (Turkey). J Biodivers Environ Sci. 2007;1(1):15-22.

119. Yavuz M, Çobanoğlu G. Lichen flora of Pamukkale (Hierapolis), Turkey. Pakistan Journal of Biological Sciences. 2007;10(17):2998-3001. https://doi.org/10.3923/pjbs.2007.2998.3001

120. Kınalığlu K. Floristic lichen records from Uşak Province, Turkey. International Journal of Botany. 2008;4(4):444-449. https://doi.org/10.3923/ijb.2008.444.449

121. Tufan-Çetin Ö, Sumbul H. Lichens of the Köprülü Canyon National Park in Turkey. Mycotaxon. 2011;115:534-536.

122. Kinalioğlu K, Aptroot A. Some lichens from Afyonkarahisar and Kırıkkale provinces. Gazi University Journal of Science. 2012;25(2):301-306.

123. Yazici K, Aptrool A, Aslan A. The lichen biota of Iğdır province (Turkey). Mycotaxon. 2013;123:492.

124. Berger F, Türk R. Die Flechtenflora im unteren rannatal (Mühlviertel, Oberösterreich, Österreich). Beiträge zur Naturkunde Oberösterreichs. 1995;3:147-216.

125. Hafellner J. Ein Beitrag zur Flechtenflora des Jogllandes (Steiermark). Mitteilungen des Naturwissenschaftlichen Vereines für Steiermark. 2003;133:81-97.

126. Coste C. Inventaire raisonné des lichens et des champignons lichénicoles de la forêt domaniale de Grésigne (tarn, France). Bulletin de la Société d'Histoire Naturelle de Toulouse. 2012;148:3-12.

127. Hafellner J, Obermayer S, Obermayer W. Zur Flechtendiversität in den Gurktaler Alpen (Österreich: Kärnten, Steiermark und Salzburg). Herzogia. 2005;18:79-138.

128. Motiejūnaitè J. Lichens of neglected habitats in Eastern and East-Central European lowlands. Acta Mycol. 2006;41(1):145-154. https://doi.org/10.5586/am.2006.018

129. Ardelean IV, Keller C, Scheidegger C. Lichen flora of Rodnei Mountains National Park (Eastern Carpathians, Romania) including new records for the Romanian mycoflora. Folia Cryptogam Est. 2013;50:101-115. https://doi.org/10.12697/fce.2013.50.13

130. Bilovitz PO, Mayrhofer H. A contribution to the lichenized fungi of Serbia. Sauteria. 2008;15:79-94.

131. Motiejūnaite J, Stončius D, Dolnik C, Tõrra T, Uselinė A. New and noteworthy for Lithuania lichens and lichenicolous fungi. Bot Lith. 2007;13(1):19-25.

132. Randlane T, Jüriado I, Suija A, Lõhmus P, Leppik E. Lichens in the new red list of Estonia. Folia Cryptogam Est. 2008;44:113-120.

133. van den Boom PPG. Contribution to the knowledge of lichenicolous fungi and lichens from Portugal and Spain. Osterr Z Pilzkd. 2000;9:151-162.

134. Crespo A, Barreno E, Rico VJ, Bueno AG. Catálogo liquénico del desierto de Calanda (Teruel, España). I. An Jard Bot Madr. 1980;36:43-55.

135. Vonarburg C, Cezanne R, Eichler M, Gnüchtel A, Hofmann P, Hohmamm ML, et al Artenliste der Flechten und flechtenbewohnenden Pilze im Gebiet von Finhaut (Wallis, 
Schweiz). Ergebnisse der BLAM-Exkursion 2000. Meylania. 2002;22:8-20.

136. Cansaran Duman D, Aras S, Atakol O. Determination of usnic acid content in some lichen species found in Anatolia. Journal of Applied Biological Sciences. 2008;2(3):41-44.

137. Mayer W, Turk R. Flechten in Kulturlandschaften III - Steyr und Umgebung (Oberösterreich, Austria). Beiträge zur Naturkunde Oberösterreichs. 2002;11:83-140.

138. Halda JP. Interesting lichen records from Králický Sněžník Mts. (Glatzer Schneeberg, Czech Republic). In: Lackovičová A, Guttová A, Lisická E, Lizoň P, editors. Central European lichens - diversity and threat. Ithaca, NY: Mycotaxon; 2006. p. 315-324.

139. Josef P, Halda JP. Seznam lišejníků české strany Králického Sněžníku. Acta Musei Richnoviensis, Section Nature. 2008;15(2):43-84.

140. Schiefelbein U, Ratzel S. Beitrage zur Flechtenflora Mecklenburg-Vorpommerns (Deutschland) und angrenzender Gebiete. Herzogia. 2005;18:63-77.

141. Viktoria N, Tarasova L, Angella V, Androsova SV, Ahti T. The present lichen flora of the city of Petrozavodsk. Folia Cryptogam Est. 2013;50:57-66. https://doi.org/10.12697/fce.2013.50.08

142. Gulnara M, Tagirdzhanova O, Kataeva A, Stepanchikova I. New lichen records from the Novgorod region, Russia. Folia Cryptogam Est. 2014;51:103-107. https://doi.org/10.12697/fce.2014.51.11

143. Alstrup V. Additions to the lichens and lichenicolous fungi of the Syktyvkar area, Komi Republic, Russia. Graph Scr. 2014;26(1-2):40-41.

144. Paukov AG, Teptina AY. New records of lichens from Middle Urals, Russia. Folia Cryptogam Est. 2012;49:39-43.

145. Aragón G, Sarrión FJ, Martínez I. Epiphytic lichens on Juniperus oxycedrus L. in the Iberian Peninsula. Nova Hedwigia. 2004;78:45-56. https://doi.org/10.1127/0029-5035/2004/0078-0045

146. Aragón G, Rico VJ, Belinchón R. Lichen diversity from Cazorla, Segura and Las Villas Biosphere Reserve (SE Spain). Nova Hedwigia. 2006;82:31-50. https://doi.org/10.1127/0029-5035/2006/0082-0031

147. Svensson M, Palice Z. Additions to the montane lichen flora of Sweden. Graph Scr. 2009;21:23-32.

148. Spinelli A, Mermillod JC, Vust M. Die Artenvielfalt der Flechten im Münstertal. Jahresbericht Natf Gesundheitsamt Graubünden. 2012;117:19-32.

149. Candan M, Türk AÖ. Lichens of Malatya, Elazığ and Adıyaman provinces (Turkey). Mycotaxon. 2008;105:19-22.

150. Coppins BJ, Kondratyuk SYa, Khodosovtsev AY, Zelenko SD, Wolseley PA. Contribution to lichen flora of Ukrainian Carpathians. Chornomors'kyi Botanical Journal. 2005;1(2):5-23. 